
Not for reproduction, distribution or commercial use.

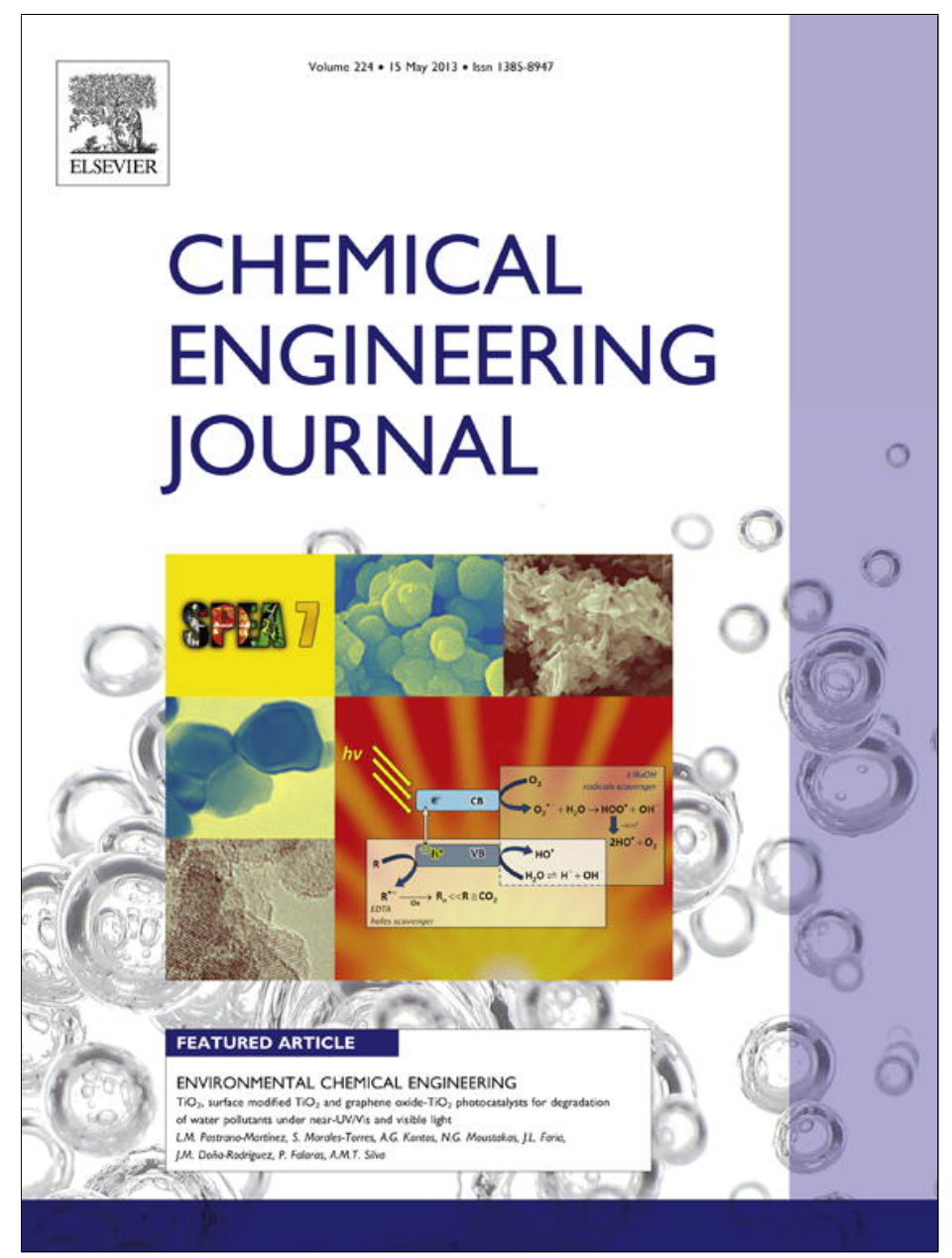

This article appeared in a journal published by Elsevier. The attached copy is furnished to the author for internal non-commercial research and education use, including for instruction at the authors institution and sharing with colleagues.

Other uses, including reproduction and distribution, or selling or licensing copies, or posting to personal, institutional or third party websites are prohibited.

In most cases authors are permitted to post their version of the article (e.g. in Word or Tex form) to their personal website or institutional repository. Authors requiring further information regarding Elsevier's archiving and manuscript policies are encouraged to visit: 


\title{
A pervaporation photocatalytic reactor for the green synthesis of vanillin
}

\author{
G. Camera-Roda ${ }^{\mathrm{a}, *}$, V. Augugliaro ${ }^{\mathrm{b}}$, A. Cardillo $^{\mathrm{a}}$, V. Loddo $^{\mathrm{b}}$, G. Palmisano $^{\mathrm{b}}$, L. Palmisano $^{\mathrm{b}}$ \\ ${ }^{a}$ Dipartimento di Ingegneria Chimica, University of Bologna, Via Terracini 28, 40131 Bologna, Italy \\ b "Schiavello-Grillone" Photocatalysis Group, Dipartimento di Ingegneria Elettrica Elettronica e delle Telecomunicazioni, di tecnologie Chimiche, \\ Automatica e modelli Matematici (DIEETCAM), University of Palermo, Viale delle Scienze, 90128 Palermo, Italy
}

\section{H I G H L I G H T S}

- Photocatalysis and pervaporation can be straightforwardly coupled in an integrated "green" process to produce vanillin.

- Vanillin is recovered by pervaporation while it is produced to prevent its oxidative degradation.

- The yield of the process is highly enhanced.

- The permeate is enriched in vanillin and is absolutely free of the photocatalytic fine particles.

- The ratio of the characteristic rate of pervaporation to the one of reaction is a key parameter.

\section{A R T I C L E I N F O}

\section{Article history:}

Available online 2 November 2012

\section{Keywords:}

Photocatalysis

Pervaporation

Membrane reactor

Integrated process

Green chemistry

Vanillin

\section{G R A P H I C A L A B S T R A C T}

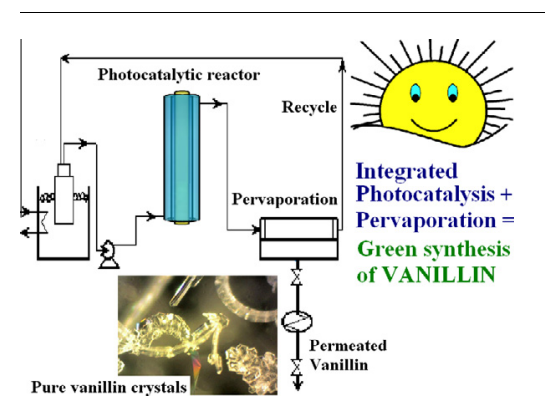

\begin{abstract}
A B S T R A C T
Pervaporation is the ideal membrane separation process to be coupled with photocatalysis for the green synthesis of vanillin from ferulic acid. In fact the operative conditions of the two processes are absolutely congruent and the integration is straightforward even operating with separate equipments. The utilization of a highly selective membrane allows the continuous recovery of vanillin by pervaporation from the reacting solution, so that its oxidative degradation is largely avoided and the yield is substantially enhanced. The effects of the main parameters are analyzed resorting to a mathematical model, which is validated by a comparison with the experimental data. In particular it is demonstrated that an optimal value of the ratio of the rate of pervaporation to the rate of reaction maximizes the "intensification" of the process. Other benefits of the integrated process, such as the complete retention of the photocatalytic powder in the reacting solution and high degree of purification of the product are presented and discussed.
\end{abstract}

(c) 2012 Elsevier B.V. All rights reserved.

\section{Introduction}

Vanillin is probably the most important aroma with an annual world production of about $12,000 \mathrm{t} / \mathrm{y}$ [1-5] and it is widely utilized as a flavoring or functional ingredient in food, cosmetic, pharmaceutical and nutraceutical products [6]. As a matter of fact, the pricey natural extract produced from vanilla pods can satisfy only a very small percentage (less than 1\%) of the demand [7] and both

\footnotetext{
* Corresponding author. Tel.: +39 0512090239; fax: +39 0512090247.

E-mail address: giovanni.cameraroda@unibo.it (G. Camera-Roda).
}

the yearly production and the market of the natural vanillin are very unstable, so it is unavoidable to resort to production alternatives [8].

Currently this aromatic aldehyde is mostly synthesized by chemical routes from lignosulfonates of the paper and pulp industry or from guaiacol of petrochemical derivation and it is sold at a price which is approximately two orders of magnitude lower than the one of the natural product. However, nowadays, the need of conservation of world resources encourages both sustainability and green chemistry, which are not achieved by the classical synthetic routes $[8,9]$. Furthermore the final consumers of these type 
of goods (food, cosmetics, drugs and nutraceutics) are becoming particularly interested in green products [10-13], so a green production route may have a strategic importance for the marketing of the final product. Indeed Rhovanil Natural, the biotechnologically produced vanillin, which according to the US and EU rules can be labeled "natural", is industrially produced by Rhodia and, even if its price is much higher than the one of the "nature-identical" vanillin (the chemically synthesized product), it has been satisfactorily accepted by the market [14]. So, the biotechnological route has been the subject of many studies (see e.g. [15-22]), but till now only the commercial biotech product [23] by Rhodia is present on the market. Actually, the biotechnological way is limited by the high costs, which are a consequence of the accurate control of the process, of the inhibition by the chemicals, of the degradation of the product, of the necessity of selected strains of microorganisms and of the complex purification.

All these difficulties motivate the search for an alternative eco-friendly synthesis of vanillin. Recently Augugliaro et al. [24] proposed a photocatalytic synthesis of vanillin, which encompasses the requirements of green chemistry. They demonstrated that vanillin is an intermediate compound obtained by the photocatalytic reaction of some precursors, which can be also of natural origin. However, as an intermediate compound, vanillin is further degraded into other chemicals at a non-negligible rate, so that the selectivity is negatively affected. On the other hand, CameraRoda et al. [25] demonstrated that the yield of some aromatic aldehydes, produced by the photocatalytic partial oxidation of the corresponding alcohols, can be significantly enhanced by coupling the reaction with a membrane separation in a pervaporation photocatalytic reactor (PVPR), so that the produced aldehyde can be recovered while it is formed to prevent its degradation. By the way, this coupling brings also other important benefits [25]. The process enhancement that can be obtained with a PVPR largely depends on the membrane selectivity towards the products with respect to the reactants. When the reactants and the products are chemically similar, as it is the case for aldehydes produced by partial oxidation, this conditions must be accurately verified, since in pervaporation the separation performances are determined by the physico-chemical properties of the chemicals, in particular by the solubility and the diffusivity of the permeants in the membrane [26,27].

In the biotechnological production of vanillin, the utilization of pervaporation to remove vanillin from the fermentation broth has already been proposed $[28,29]$, with the aim of reducing the inhibition effect of vanillin on the biological reaction. However, the possibility of biofouling on the membrane surface and the low permeate flux, which is obtained at the temperatures which are compatible with the fermentation, limit the efficacy of an integrated process and make realistic the utilization of pervaporation only for a post process separation or purification. Generally, these limitations are much less severe when pervaporation is coupled with photocatalysis [25,30]. Therefore, it appears interesting to investigate the feasibility of the integration of photocatalysis and pervaporation in a PVPR to enhance the yield of the produced vanillin.

\section{Materials and methods}

\subsection{Experimental}

All the chemicals (vanillin, trans-ferulic acid, and standards for caffeic acid, vanillylmandelic acid, homovanillic acid, vanillic acid) were purchased from Sigma-Aldrich with a purity $>99 \%$. Standards for cis-ferulic acid were obtained by partially transforming known quantities of trans-ferulic acid under UVA irradiation in controlled conditions [31]. Commercial $\mathrm{TiO}_{2}$ Merck (100\% anatase) was chosen in view of its favorable selectivity in the production of vanillin [24] and was used as received.

The reacting solution was prepared by dissolving ferulic acid in deionized water at $60{ }^{\circ} \mathrm{C}$ overnight. Merck $\mathrm{TiO}_{2}$ powder was suspended in this solution and ultrasonicated for $15 \mathrm{~min}$ just before the experiments with the slurry photocatalytic reactor. Additional tests established that the decrease of the concentration of ferulic acid due to dark reaction and to adsorption is negligible.

The set up of the experimental apparatus is shown in Fig. 1.

The fluid passes through an annular photocatalytic reactor and a thermostated pervaporation cell, where the permeate is separated from the retentate. This latter enters a flask immersed in a thermostatic bath before being recycled by a peristaltic pump to the reactor. The permeated vapors are partly condensed in a first vial at $12{ }^{\circ} \mathrm{C}$, then the remaining fraction in a second vial immersed in a liquid nitrogen trap. The tube which connects the pervaporation module to the condensation system is electrically heated to prevent condensation.

The permeate pressure is kept at approximately 2-3 mbar with the aid of a vacuum pump. It must be observed that the operation mode is semicontinuous since, even if no feed stream is present, the permeate is continuously collected by condensation.

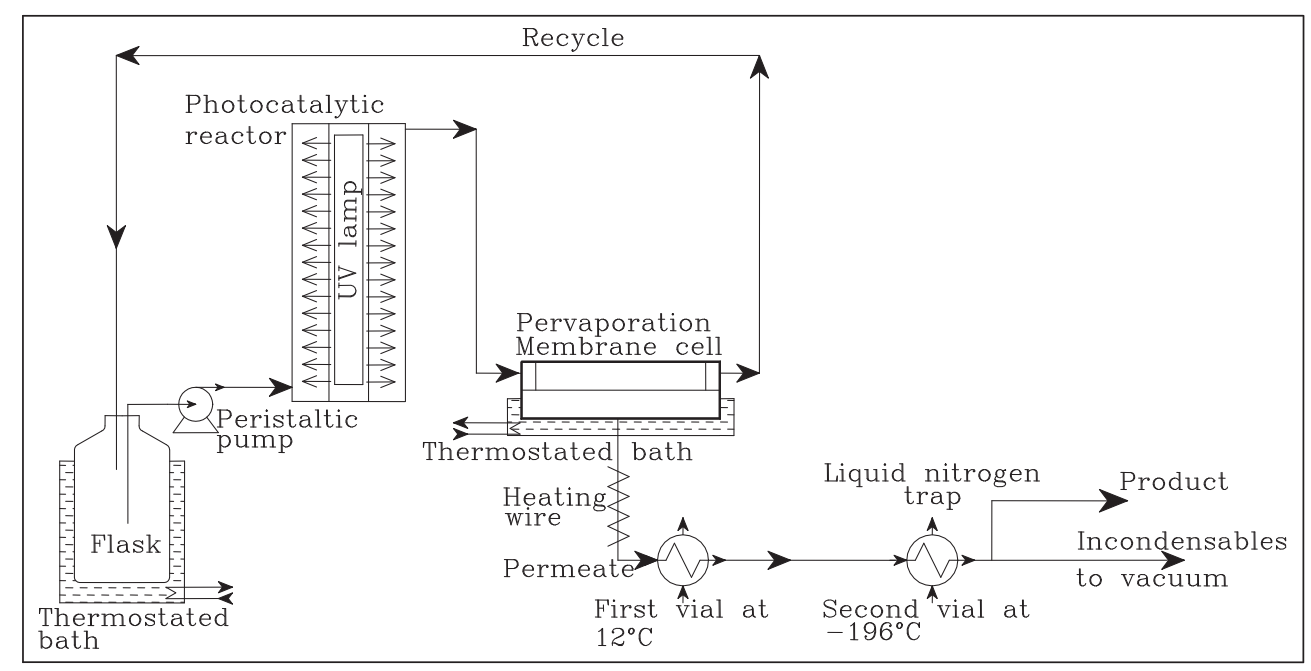

Fig. 1. Schematic representation of the experimental apparatus. 
The coupling between photocatalysis and pervaporation takes place thanks to the recycle loop. All the tubings are in PTFE or in Masterflex Chem-Durance ${ }^{\circledR}$ (for the peristaltic pump) to avoid the adsorption of the organic compounds.

Photocatalytic (without pervaporation) and pervaporation (without photocatalysis) experiments were carried out with the same apparatus, simply eliminating the unnecessary operation from the recycle loop.

The samples, which are withdrawn from the reacting solution at different times, and the collected permeate were analyzed by a Shimadzu LC-20A HPLC system equipped with a $3 \mu \mathrm{m}$ Dionex Acclaim PA2 column and a Diode Array Detector working at $280 \mathrm{~nm}$. The eluent is a mixture of water, acetic acid and acetonitrile (85:10:5 volumetric ratios) with a flow rate of $0.5 \mathrm{~mL} \mathrm{~min}^{-1}$.

The dimensions of the annular photoreactor are: inner diameter $=2.4 \mathrm{~cm}$; outer diameter $=3.4 \mathrm{~cm}$ and length $=26.5 \mathrm{~cm}$. On the axis of the reactor a linear fluorescent blacklight lamp (Philips $8 \mathrm{~W} \mathrm{TL} / 08$ ) emits UVA radiation from 350 to $400 \mathrm{~nm}$ with $82 \%$ of the emitted radiant power concentrated from 360 to $380 \mathrm{~nm}$ and an emission maximum at $365 \mathrm{~nm}$. Glass walls in Pyrex minimize the absorption of the UVA radiation. A radiation intensity of about $24 \mathrm{~W} \mathrm{~m}^{-2}$ was measured by a Delta Ohm HD9021 radiometer with a LP9021 UVA probe at the inner radius of the annulus.

A pervaporation cell with an outward radial flow flowing on the membrane was used. The diameter of the part of the membrane exposed to pervaporation is $7.45 \mathrm{~cm}$ for a permeating surface area of $44 \mathrm{~cm}^{2}$. The membrane was prepared by dissolving PEBAX ${ }^{\circledR}$ 2533 (polyether block amide by Arkema) in n-butyl alcohol and isopropyl alcohol (PEBAX $8.7 \mathrm{w} \%$, n-butyl alcohol $22.8 \mathrm{w} \%$, isopropyl alcohol $68.5 \mathrm{w} \%$ ), casting the solution onto a glass plate by a doctor blade with adjustable clearance and evaporating the solvents for 1 day at $70^{\circ} \mathrm{C}$. The thickness of the dry membrane is about $45 \mu \mathrm{m}$.

Some preliminary investigations were also made with POMS/ PEI membranes (polyoctylmethylsiloxane on polyetherimide by GKSS) but PEBAX membranes were finally chosen since they showed to be better performing than POMS/PEI membranes (in particular for the permeate flux of vanillin), as it is established also through the comparison of the results by Böddeker with PEBAX [28] with those by Brazinha with POMS/PEI [29].

Among the possible precursors (or substrates according to the usual terminology of photocatalysis) for the production of vanillin, ferulic acid was chosen since it can be of natural origin, it is sufficiently soluble into water and the selectivity to vanillin is satisfactory [24]. In all the experiments of the present investigation the initial concentration of trans-ferulic acid was $0.5 \mathrm{mM}$.

\subsection{Choice of the operative conditions}

When pervaporation and photocatalysis are carried out in distinct equipments, an effective integration can be obtained by interconnecting them by a recycle with a sufficiently high flow rate, which must guarantee that the ratio between the characteristic time of disappearance of the substrate from the system and the residence time in the photoreactor-pervaporation module is much larger than $1[27,30]$. The adopted recycle flow rate of $61 \mathrm{~L} \mathrm{~h}^{-1}$ satisfies this condition since this ratio is greater than $10^{3}$. On the other hand, the utilization of separate equipments avoids some possible incompatibility of the two processes and allows the optimization of the design of the specific apparatus for the relevant process. For instance it has been observed that the life of the membrane is significantly shortened when it is exposed to the UVA radiation in presence of the photocatalyst, so the pervaporation membrane has been kept separated from the reactor. Nonetheless, it is advisable that the operative conditions of the two processes, such as temperature, pressure and concentration of chemicals are

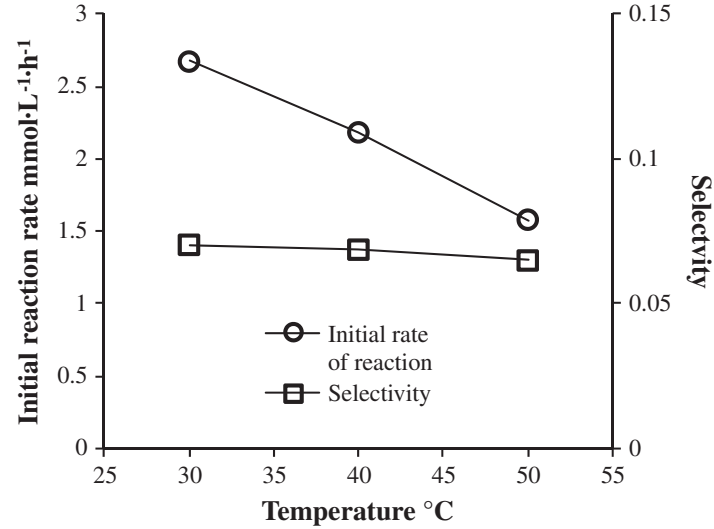

Fig. 2. Effect of the temperature on the initial rate of reaction and on the reaction selectivity at $15 \%$ of conversion.

congruent. Pervaporation and photocatalysis are perfectly compatible for the last two conditions, since atmospheric pressure and diluted aqueous solutions represent the normal working conditions both in photocatalysis and in organophilic pervaporation. In pervaporation relatively high temperatures favor the permeability especially for high boiling compounds, such as vanillin $[28,32]$. The effect of the temperature on the initial rate of photocatalytic reaction and on the reaction selectivity for the synthesis of vanillin has been studied in photocatalytic experiments without pervaporation. The obtained results are shown in Fig. 2.

The initial rate of reaction decreases to some extent with the temperature, probably due to the reduced adsorption of the reagents and to the diminution of the dissolved oxygen concentration, whereas a negligible effect of the temperature is observed for the reaction selectivity. The negative effect of the increase of the temperature on the reaction rate is largely counterbalanced by the enhancement of the permeate flux, which in the pervaporation experiments carried out at $0.1 \mathrm{mM}$ feed concentration of vanillin showed to increase with a relatively high activation energy (approximately $58 \mathrm{~kJ} \mathrm{~mol}^{-1}$ ). Therefore it has been chosen to work the experiments at $50^{\circ} \mathrm{C}$, a temperature high enough to obtain a satisfactory permeate flux without severely affecting the rate of reaction. Furthermore, at this temperature a more favorable value (closer to the optimal value, which will be discussed later on) of the ratio of the characteristic rate of permeation to the characteristic rate of reaction (a key parameter for PVPRs) can be obtained. At $50{ }^{\circ} \mathrm{C}$ the measured permeate flux is about $390 \mathrm{~g} \mathrm{~h}^{-1} \mathrm{~m}^{-2}$ with an enrichment factor for vanillin, $\beta_{\mathrm{van}}$, defined as the ratio of its concentration in the permeate to the one upstream the membrane, equal to 4.2. Both the enrichment factor and the flux remained almost constant during the process.

The concentration of the photocatalyst is $0.43 \mathrm{~g} \mathrm{~L}^{-1}$ in order to get an optical thickness of about 6 , which permits both a satisfactory absorption of the radiation and a sufficiently effective exploitation of the reactor volume [33].

\subsection{Mathematical model}

In the photocatalytic production of vanillin the reaction pathways are very complex with a high number of parallel reactions which give different intermediates and then final compounds, possibly till complete mineralization [24]. Fig. 3 illustrates the scheme of the reactions involving the original substrate (ferulic acid) and the desired product (vanillin). Other different reactions that finally lead to the mineralization of other intermediates are not indicated in Fig. 3, since they can be disregarded for the present model. 

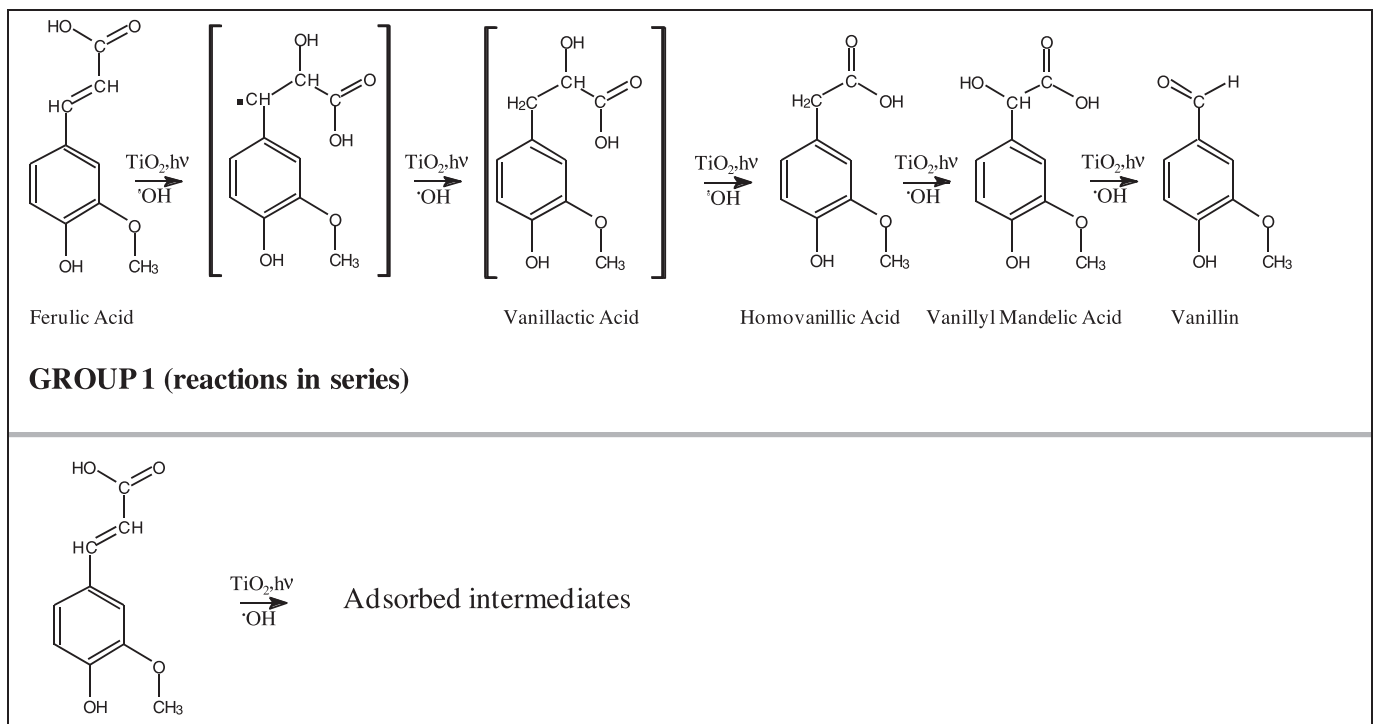

Ferulic Acid
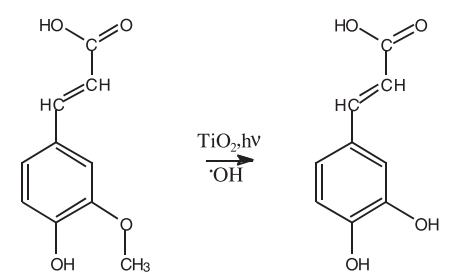

Ferulic Acid

trans-Caffeic Acid<smiles>COc1cc(/C=C/C(=O)O)ccc1O</smiles>

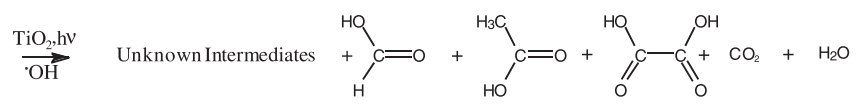

Ferulic Acid

GROUP 2 (parallel reactions)
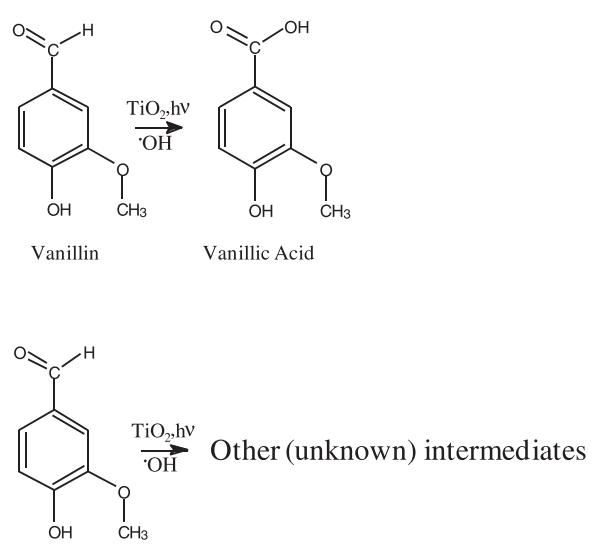

Vanillin

GROUP 3 (parallel reactions)

Fig. 3. Scheme of the reactions involving vanillin and ferulic acid. 
In spite of the very complex reaction pathway, the following proposed simplified model can reproduce with good accuracy the time evolution of the compounds of main interest: the substrate (ferulic acid) and the desired product (vanillin). Firstly, it must be observed that the initial substrate (trans-ferulic acid) is partially transformed into cis-ferulic acid when irradiated by UVA radiation. However, photocatalytic experiments, not reported here for brevity, show that there is no substantial difference in the (photocatalytic) reactivity of these two isomers, so, in the following development, "ferulic acid" will refer comprehensively to both of the isomeric forms.

Referring to Fig. 3, the reactions of interest for the substrate, $\mathrm{S}$ (ferulic acid), and for the product, $\mathrm{P}$ (vanillin), can be summarized as:

Group (1) $S \rightarrow I_{1} \rightarrow \cdots \rightarrow I_{k-1} \rightarrow I_{k} \rightarrow I_{k+1} \rightarrow \cdots I_{K} \rightarrow P$

Group 1 of reactions in series with $k=1, \ldots, K$, where $I_{k}$ are intermediate compounds, leads to the formation of P. Since the concentration of these intermediates in the reacting solution remains always very low, the rate of formation of $P$ can be assumed to be limited by the rate of the first reaction. It is also likely that the rates of the possible different parallel reactions which transform the intermediate compounds $I_{k}$ are negligible.

(Group (2) $S \rightarrow B_{i}$

Group 2 of reactions in parallel with $i=2, \ldots, N$, where $N$ is the number of parallel reactions $(i=1$ is not considered as it represents the reaction which produces $I_{1}$ ), transforms the substrate into intermediate products that do not produce vanillin.

(Group (3) $\quad P \rightarrow C_{j}$

Group 3 of reactions with $j=1, \ldots, M$, where $M$ is the number of parallel reactions, degrades the produced vanillin.

Other reactions which transform $B_{i}, C_{j}$ and other different produced compounds are present in the system but can be ignored for the current analysis. In view of the low concentrations of all the reactants a first order kinetics will be adopted for all the photocatalytic reactions [34].

The integration of photocatalysis and pervaporation is effective when the degree of backmixing generated by the recycle is high, as discussed in Section 2.2. In this case the fluid flowing in the reactor and in the pervaporation module is almost perfectly mixed and the mass balances in the reacting solution for $S$ and $P$ can be written as:

$\frac{d n_{S}}{d t}=-\left(k_{1} C_{S}+\sum_{i=2}^{N} k_{i} C_{S}\right) V_{R}-\frac{\dot{m}^{\prime \prime}}{\rho} A C_{S, \text { permeate }}$

$\frac{d n_{P}}{d t}=\left(k_{1} C_{S}-\sum_{j=1}^{M} k_{j} C_{P}\right) V_{R}-\frac{\dot{m}^{\prime \prime}}{\rho} A C_{P, \text { permeate }}$

where the subscripts $S$ and $P$ refer to the substrate and to the product, respectively. $n_{\mathrm{i}}$ (mmol) is the number of moles in the reacting solution, $C_{\mathrm{i}}=n_{\mathrm{i}} / V_{\mathrm{T}}\left(\mathrm{mmol} \mathrm{L}^{-1}\right)$ the concentration in the reacting solution, $\mathrm{C}_{\mathrm{i}}$, permeate $\left(\mathrm{mmol} \mathrm{L}^{-1}\right)$ the concentration in the condensed permeate, $t(\mathrm{~h})$ the time, $V_{R}(\mathrm{~L})$ the volume of the reactor, $\dot{m}^{\prime \prime}$ $\left(\mathrm{kg} \mathrm{h}^{-1} \mathrm{~m}^{-2}\right)$ the mass flux of the permeate, $\mathrm{A}\left(\mathrm{m}^{2}\right)$ the area of the membrane, $\rho\left(\mathrm{kg} \mathrm{L}^{-1}\right)$ the density of the condensed permeate. $V_{\mathrm{T}}$ represents the total volume of the solution and can be calculated as $V_{T}=V_{T 0}-\frac{\dot{m}^{\prime \prime}}{\rho} A t$ where $V_{\mathrm{TO}}$ is the total volume at $t=0$. The first term at the right hand side of Eqs. (1) and (2) represents the molar rate of formation (or disappearance if negative) due to the photocatalytic reactions. The second term represents the molar rate of removal by pervaporation. $k_{1}, k_{\mathrm{i}}$ and $k_{\mathrm{j}}\left(\mathrm{h}^{-1}\right)$ are apparent first order kinetic constants. They depend on the space distribution of the local volumetric rate of radiant energy absorption (LVREA) inside the reactor. This space distribution is obviously unique for a given reactor at a given time [27].

Eqs. (1) and (2) can be rearranged as:

$\frac{d n_{S}}{d t}=-\left[\left(1+R_{2}\right) k_{1} V_{R}+\frac{\dot{m}^{\prime \prime}}{\rho} A \beta_{S}\right] C_{S}$

$\frac{d n_{P}}{d t}=k_{1} C_{S} V_{R}-\left[k_{1} R_{3} V_{R}+\frac{\dot{m}^{\prime \prime}}{\rho} A \beta_{P}\right] C_{P}$

where $R_{2}=\frac{\sum_{i=2}^{N} k_{i}}{k_{1}}, R_{3}=\frac{\sum_{j=1}^{M} k_{j}}{k_{1}}$ and $\beta_{\mathrm{i}}$ is the enrichment factor, which is defined as the ratio of the concentration in the condensed permeate to the one upstream the membrane $\left(\beta_{i}=C_{i, \text { permeate }} / C_{i}\right)$.

The apparent kinetic constants may vary with time due to several phenomena, such as agglomeration of the photocatalytic particles, deposition of the particles on the surfaces of tubes and apparatuses, absorption of the UVA radiation by some products and competition of the intermediates for the utilization of photogenerated oxidizing agents or of the active photocatalytic sites. Since it is rather complex to quantify these effects, $k_{1}(t)$ is evaluated by fitting the experimental time evolution of the number of moles of $\mathrm{S}$, whereas the ratios, $R_{2}$ and $R_{3}$, between apparent kinetic constants are anyway assumed to be constant, since it is likely that the aforementioned phenomena may affect at the same extent the rates of all the photocatalytic reactions. The experimental results of photocatalytic runs without pervaporation have been utilized to evaluate $R_{2}$ and $R_{3}$. Specifically, taking into account Eqs. (1) and (2), the initial rate data have been utilized to determine $R_{2}$ as $R_{2}=\frac{\left(-\left.\frac{d n_{S}}{d t}\right|_{t=0}\right)-\left(\left.\frac{d n_{p}}{d t}\right|_{t=0}\right)}{\frac{d n_{P}}{d t} t_{t=0}}$, whereas $R_{3}$ has been computed by fitting the experimental values of $n_{P}$ vs. time with the least squares method.

For the permeate, the following mass balances are used to calculate the cumulative number of moles of ferulic acid, $n_{S \text {, permeate, }}$ and vanillin, $n_{P, \text { permeate, }}$ collected in the permeate:

$\frac{d n_{S, \text { permeate }}}{d t}=\frac{\dot{m}^{\prime \prime}}{\rho} A \beta_{S} C_{S}$

$\frac{d n_{P, \text { permeate }}}{d t}=\frac{\dot{m}^{\prime \prime}}{\rho} A \beta_{P} C_{P}$

The initial conditions, at $t=0$, are: $n_{\mathrm{S}}=n_{S 0}, n_{P}=0$, and $n_{S, \text { permeate }}=\mathrm{n}_{P, \text { permeate }}=0$.

Once the differential Eqs. (3)-(6) are solved, the conversion, $\chi$, the selectivity, $\sigma$, and the yield, $\eta$, can be computed as:

$$
\begin{gathered}
\chi=\frac{n_{S 0}-\left(n_{S}+n_{S, \text { permeate }}\right)}{n_{S 0}} ; \quad \sigma=\frac{n_{P}+n_{P, \text { permeate }}}{n_{S 0}-\left(n_{S}+n_{S, \text { permeate }}\right)} ; \\
\eta=\chi \cdot \sigma=\frac{n_{P}+n_{P, \text { permeate }}}{n_{S 0}}
\end{gathered}
$$

In the case at constant $k_{1}$, the solution of the previous ODEs is analytical. On the contrary, when the experimental time-variation of $k_{1}$ is taken into account, the ODEs have to be solved numerically. The ODE version of the Crank-Nicolson method with a $0.1 \mathrm{~h}$ constant time interval has been used to this aim.

From independent measurements the following values of the parameters were obtained and have been used in the simulations of the PVPR:

$V_{R}=0.12 \mathrm{~L}, \quad V_{\mathrm{T} 0}=0.6 \mathrm{~L}, \quad R_{2}=6.8, \quad R_{3}=8.7, \quad \rho=1 \mathrm{~kg} \mathrm{~L}^{-1}, \quad A=$ $0.44 \cdot 10^{-2} \mathrm{~m}^{2}, \dot{m}^{\prime \prime}=0.39 \mathrm{~kg} \mathrm{~h}^{-1} \mathrm{~m}^{-2}, \beta_{S}=0.005, \beta_{P}=4.2$.

In view of the fact that $R_{3}$ is significantly greater than 1 , the kinetic of degradation of vanillin is noteworthy, so that the convenience of recovering vanillin is high [35].

Through a dimensional analysis, the intervening dimensionless parameters turn out to be: $R_{2}=6.8, R_{3}=8.7, R_{V}=V_{\mathrm{TO}} / V_{R}=5, \beta_{\mathrm{S}}=$ $0.005, \beta_{P}=4.2, \delta=\frac{\left(\dot{m}_{p}^{\prime \prime} / \rho\right)}{k_{1}, a v} \cdot \frac{A}{V_{R}}=0.254$, 
$\delta$ representing the ratio of the characteristic rate of permeation to the characteristic average rate of reaction $[27,30,36]$.

\section{Results}

In pervaporation experiments with a $0.1 \mathrm{mM}$ aqueous solution of vanillin at $50{ }^{\circ} \mathrm{C}$, the measured total permeate flux is about $0.390 \mathrm{~kg} \mathrm{~h}^{-1} \mathrm{~m}^{-2}$ with an enrichment factor $\beta_{\text {van }}$ for vanillin equal to 4.2. Since the value of the enrichment factor is higher than one, then the permeate results to be "enriched" with vanillin. Thanks to the high dilution of the organic permeants in the aqueous solution both the enrichment factor and the flux do not change appreciably in the integrated process when also the substrate and other photocatalytically produced compounds are present.

The capability of the membrane to selectively recover vanillin and, at the same time, to largely retain the substrate in the reactor is essential to get a process intensification with the integrated process [30]. The chromatograms of the reacting solution after $2 \mathrm{~h}$ in the integrated process and of the corresponding permeate are reported in Fig. 4.

The chromatograms in Fig. 4 show that only vanillin, and of course water, permeate at a non-negligible extent. Therefore, with
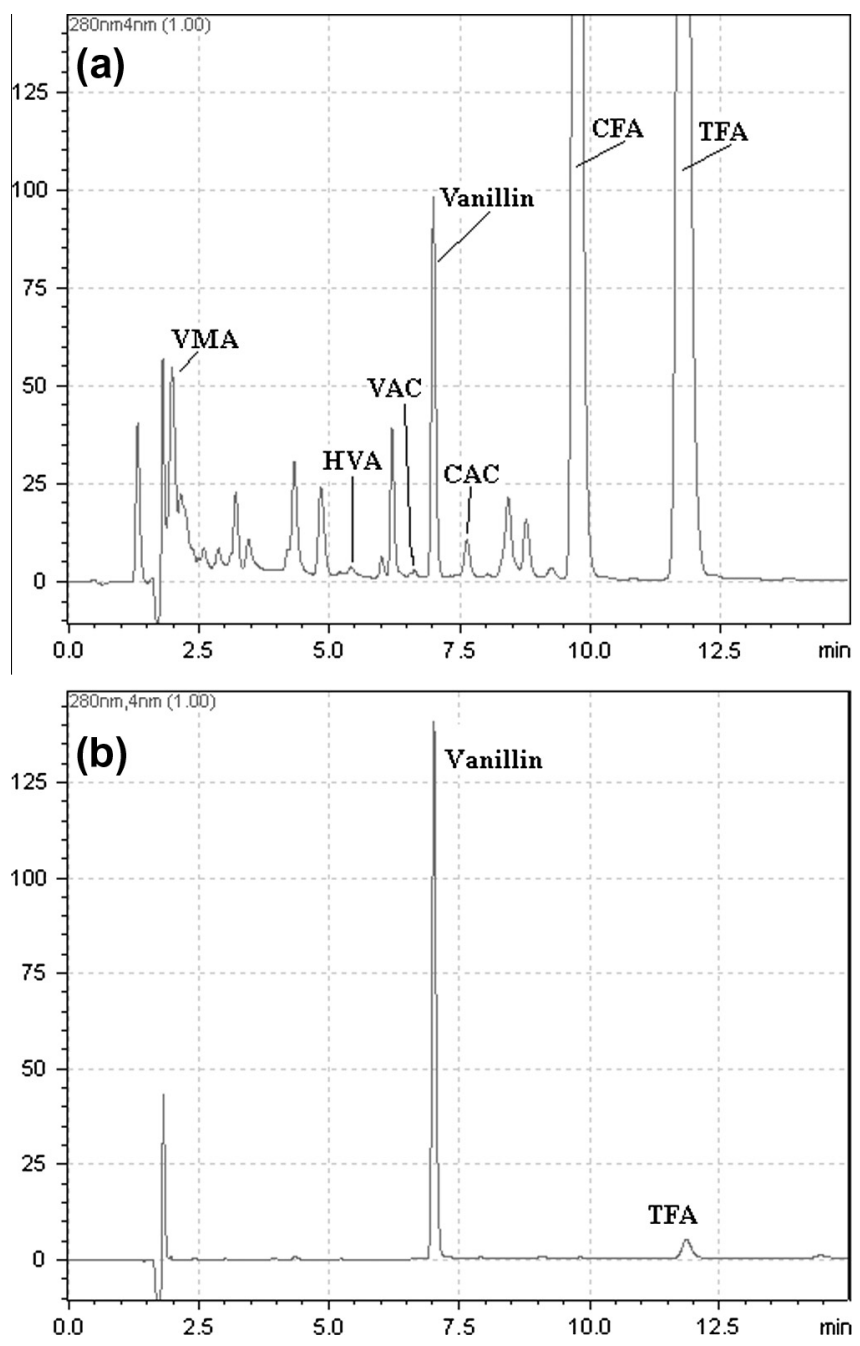

Fig. 4. HPLC chromatograms of: (a) the reacting solution at $t=2 \mathrm{~h}$; (b) the permeate at the same time. VMA: vanillylmandelic acid; HVA: homovanillic acid; VAC: vanillic acid; CAC: caffeic acid; CFA: Cis-ferulic acid; TFA: trans-ferulic acid; other peaks are unknown. the exception of vanillin and water, the retention is extraordinarily high for all the other known and unknown compounds, which are photocatalytically produced. Actually the membrane permeates also another unknown compound, which is thermochemically produced from ferulic acid and is present at measurable concentrations in the reacting solution only at $50{ }^{\circ} \mathrm{C}$, but not at 30 and $40{ }^{\circ} \mathrm{C}$. The peak of this compound is not shown in the HPLC chromatogram since its retention time is longer than 25 min. Anyway, a further purification may be obtained by a "fractional condensation" of the vapors of vanillin $[28,29]$, which deposit as pure crystals in the vial at $12^{\circ} \mathrm{C}$, provided that at this temperature the partial pressure of vanillin in the permeate is higher than approximately $1 \times 10^{-3}$ mbar.

The high rejection of ferulic acid and vanillic acid is mainly due to their dissociation into charged species at the $\mathrm{pH}$ of the reacting mixture $(\mathrm{pH} \approx 4)$ [29]. In fact, as charged species, their volatility at the membrane-permeate interface is so low that their evaporation into the permeate is very limited. Therefore, the resulting value of the "desorption factor" [37] is unfavorable to their permeation.

It must be remarked that also the photocatalytic particles are completely retained by the "dense" (non porous) membranes and this capacity allows to avoid the usually problematic post-process separation of these very fine particles from the product stream. So the permeate (product) is essentially a very purified aqueous solution of vanillin and possibly pure vanillin crystals. It is worth noting that the tendency of the "retained" photocatalytic particles to deposit on the surfaces vanishes after the first $4-5 \mathrm{~h}$, since beyond a certain thickness the deposited layers are unstable under the shear stress of the flowing fluid. Therefore, at longer times, an almost stable concentration of the photocatalyst establishes in the slurry. Furthermore, the flux decay caused by the deposition of the powders on the pervaporation membrane is absolutely negligible even after more than $20 \mathrm{~h}$ of continuous utilization. This outcome has already been observed and discussed by Camera-Roda et al. [25] for a similar PVPR.

In Fig. 5, the amount of vanillin in the reacting solution without pervaporation is compared with the one obtained in the system with the PVPR. It is evident that the simulations of the mathematical model are in good agreement with the experimental data obtained both without pervaporation and with the integrated process. The differences between the profiles in Fig. 5a and b can be ascribed to the aforementioned phenomena (agglomeration of the photocatalytic particles, deposition of the particles etc.), which contribute to vary the apparent kinetic constants, and can operate with irregular effects in the different runs.

In Fig. 5b it is apparent that the amount of vanillin cumulatively recovered by pervaporation is only a small fraction of the quantity present in the system. Considering that the performances of the membrane (flux and enrichment factor) are satisfactory, this shortage is mainly a consequence of the relatively low value of the ratio of the membrane area to the reactor volume. In effect, the estimated average value of $\delta$ obtainable in the experimental apparatus is only 0.254 , whereas it should be higher to improve the recovery of the produced vanillin and to obtain a more significant process intensification. In order to analyze the effect of $\delta$ on the yield it is opportune to use the mathematical model which has proven to simulate accurately the experimental results. It must be firstly taken into account that the yield depends also on the reaction time. In a non-integrated process it is well-known that the yield of an intermediate product of reactions in series reaches a relative maximum at an optimal reaction time (see e.g. [35]). If these reactions are carried out in a PVPR the relative maximum at an optimal reaction time is present only at low values of $\delta$, as long as the degree of the recovery of vanillin by pervaporation is inadequate. At higher value of $\delta$ the relative maximum disappears and the yield becomes monotonically increasing with the reaction time, since a larger 

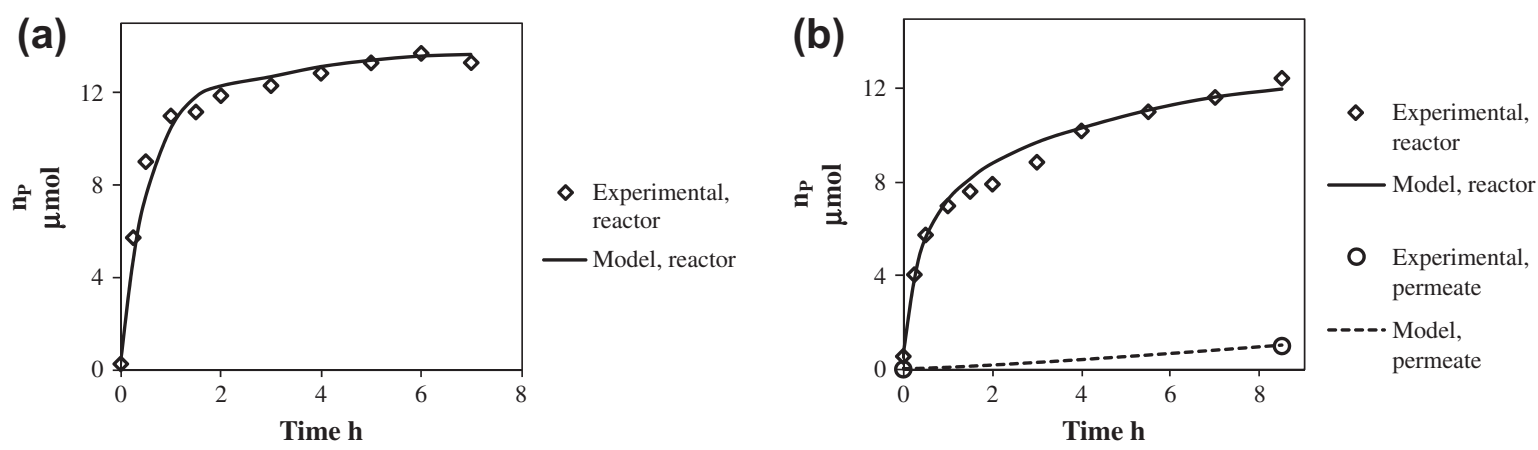

Fig. 5. The molar amount of vanillin vs. time: (a) in the reacting solution without pervaporation; (b) in the reacting solution and cumulatively recovered in the permeate with the PVPR.

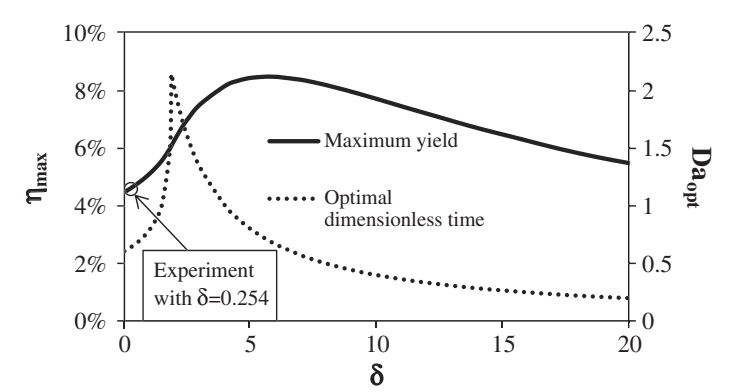

Fig. 6. Maximum yield $\left(\eta_{\max }\right)$ and optimal dimensionless reaction time $\left(\mathrm{Da}_{\mathrm{opt}}\right) \mathrm{vs.} \delta$ for $R_{2}=6.8, R_{3}=8.7, R_{\mathrm{V}}=5, \beta_{\mathrm{S}}=0.001, \beta_{\mathrm{P}}=4.2$. The arrow indicates the maximum yield for the presented experiment.

fraction of the produced vanillin is recovered by pervaporation and therefore the fraction of vanillin degraded by the consecutive reaction decreases. Note that the reaction time in a semicontinuos PVPR, of the type here investigated, cannot exceed a given finite value, since it is limited by the depletion of the reacting solution caused primarily by the permeation of water. Therefore, at relatively high values of $\delta$, the "optimal reaction time" becomes this limit value, nonetheless it is usually sufficient an appreciably shorter time to obtain a yield sufficiently close to the maximum.

In the following simulation it has been assumed that $k_{1}$ does not vary with the time. Fig. 6 shows the effects of $\delta$ on the maximum value of the yield, $\eta_{\max }$, and the corresponding optimal dimensionless time, which will be here conveniently expressed as an optimal Damköhler number, $\mathrm{Da}_{\text {opt }}$. In fact, in a reacting system the Damköhler number (presently defined as $\mathrm{Da}=\mathrm{t} \times \mathrm{k}_{1}$ ) represents the ratio of the time to a characteristic time of reaction (in this case $k_{1}^{-1}$ ) and therefore it can be used also to represent a dimensionless time.

The value of $\eta_{\max }$ without pervaporation, i.e. at $\delta=0$, is $4.46 \%$, whereas for the utilized PVPR (with $\delta=0.254$ ) is $4.60 \%$, confirming that in this case the enhancement given by the integrated process is not significant, since the membrane area is not sufficient to recover a satisfactory fraction of the vanillin produced by the reaction. However, for higher values of $\delta$, which can be obtained by increasing the ratio $A / V_{R}$, the recovery of the produced vanillin is more effective and consequently the yield is enhanced. An excessive increase of $\delta$ would have the negative effect to accelerate the depletion of the reacting solution, so that the reaction could not reach a satisfactory conversion. This phenomenon justifies the presence of the maximum of the curve of $\eta_{\max }$ in Fig. 5. The value of this maximum is $8.48 \%$ and it is obtained at $\delta_{\text {opt }}=5.7$ and $\mathrm{Da}_{\mathrm{opt}}=0.7$. It represents a substantial $90 \%$ enhancement with respect to the maximum yield $\left(4.46 \%\right.$ at $\delta=0$ and $\left.\mathrm{Da}_{\mathrm{opt}}=0.61\right)$ that can be obtained without pervaporation.
Note that PVPRs can be effectively utilized also in a continuous mode [27]. In this case the relevant parameters, which affect the performances, are analogous to the ones for the semicontinuous mode, but the maximum obtainable yield is higher $(11.9 \%$ for the same values of the parameters but at different optimal conditions for Da and $\delta$ ).

\section{Conclusions}

It has been demonstrated that a PVPR can be effective in increasing the yield of vanillin. The vanillin recovered in the permeate with the PVPR does not undergo any further degradation, contrary to what happens to the vanillin which remains in the photoreactor. The more important consequence is the enhancement of the selectivity and, consequently, of the yield in vanillin at any conversion of the substrate. In other words a "process intensification" can be obtained with the integration of photocatalysis and pervaporation in the PVPR. The degree of intensification is obviously affected by the values of the intervening parameters. In particular $\delta$ is a key parameter since its value depends on the ratio $A / V_{R}$ and therefore $\delta$ determines substantially the fraction of the produced vanillin which is recovered by permeation.

\section{References}

[1] M.J.W. Dignum, J. Kerler, R Verpoorte, Vanilla production: technological, chemical and biosynthetic aspects, Food Rev. Int. 17 (2001) 119-120.

[2] N.J. Walton, M.J. Mayer, A. Narbad, Vanillin, Phytochemistry 63 (2003) 505515.

[3] L. Esposito, K. Formanek, G. Kientz, F. Mauger, V. Maureaux, G. Robert, F. Truchet, Vanillin, in: Kirk-Othmer Encyclopedia of Chemical Technology, 1997, vol. 24 , pp. $812-825$.

[4] H. Korthou, R. Verpoorte, Vanilla, in: Flavours and Fragrances, Springer, 2007, pp. 203-217.

[5] S. Ramachandra Rao, G.A. Ravishankar, Vanilla flavour: production by conventional and biotechnological routes, J. Sci. Food Agric. 80 (2000) 289304.

[6] A.K. Sinha, U.K. Sharma, N. Sharma, A comprehensive review on vanilla flavor: Extraction, isolation and quantification of vanillin and others constituents, Int. J. Food Sci. Nutr. 59 (2008) 299-326.

[7] H. Priefert, J. Rabenhorst, A. Steinbüchel, Biotechnological production of vanillin, Appl. Microbiol. Biotechnol. 56 (2001) 296-314.

[8] F. Cavani, G. Centi, Sustainable development and chemistry, in. Kirk-Othmer Encyclopedia of Chemical Technology, 2011, pp. 1-61.

[9] M.B. Hocking, Vanillin: synthetic flavoring from spent sulfite liquor, J. Chem. Educ. 74 (1997) 1055-1059.

[10] P.S.J. Cheetham, Combining the technical push and the business pull for natural flavours, Adv. Biochem. Eng./Biotechnol. 55 (1997) 1-49.

[11] R.D. Straughan, J.A. Roberts, Environmental segmentation alternatives: a look at green consumer behavior in the new millennium, J. Consum. Market. 16 (1999) 558-575.

[12] M. Laroche, J. Bergeron, G. Barbaro-Forleo, Targeting consumers who are willing to pay more for environmentally friendly products, J. Consum. Market. 18 (2001) 503-520.

[13] J. Pickett-Baker, R. Ozaki, Pro-environmental products: marketing influence on consumer purchase decision, J. Consum. Market. 25 (2008) 281-293. 
[14] A.M. Rouhi, Fine chemicals firms enable flavor and fragrance industry, Chem. Eng. News 81 (2003) 54.

[15] S. Serra, C. Fuganti, E. Brenna, Biocatalytic preparation of natural flavours and fragrances, Trends Biotechnol. 23 (2005) 193-198.

[16] M.A. Longo, M.A. Sanromán, Production of food aroma compounds: Microbial and enzymatic methodologies, Food Technol. Biotechnol. 44 (2006) 335-353.

[17] A. Muheim, K. Lerch, Towards a high-yield bioconversion of ferulic acid to vanillin, Appl. Microbiol. Biotechnol. 51 (1999) 456-461.

[18] Y. Gounaris, Biotechnology for the production of essential oils, flavours and volatile isolates. A review, Flavour Frag. J. 25 (2010) 367-386.

[19] E.J. Vandamme, W. Soetaert, Bioflavours and fragrances via fermentation and biocatalysis, J. Chem. Technol. Biotechnol. 77 (2002) 1323-1332.

[20] R. Kumar, S. Singh, O.V. Singh, Bioconversion of lignocellulosic biomass: biochemical and molecular perspectives, J. Ind. Microbiol. Biotechnol. 35 (2008) 377-391.

[21] W. Schwab, R. Davidovich-Rikanati, E. Lewinsohn, Biosynthesis of plantderived flavor compounds, Plant J. 54 (2008) 712-732.

[22] R.G. Berger, Biotechnology of flavours - the next generation, Biotechnol. Lett. 31 (2009) 1651-1659.

[23] A.M. Rouhia, Fine chemicals firms enable flavor and fragrance industry, Chem. Eng. News 81 (2003) 54

[24] V. Augugliaro, G. Camera-Roda, V. Loddo, G. Palmisano, L. Palmisano, F. Parrino, M.A. Puma, Synthesis of vanillin in water by $\mathrm{TiO}_{2}$ photocatalysis, Appl. Catal. B: Environ. 111-112 (2012) 555-561.

[25] G. Camera-Roda, F. Santarelli, V. Augugliaro, V. Loddo, G. Palmisano, L. Palmisano, S. Yurdakal, Photocatalytic process intensification by coupling with pervaporation, Catal. Today 161 (2011) 209-213.

[26] K.W. Böddeker, Terminology in pervaporation, J. Membr. Sci. 51 (1990) 259272 .
[27] G. Camera-Roda, F. Santarelli, Design of a pervaporation photocatalytic reactor for process intensification, Chem. Eng. Technol. 35 (2012) 1221-1228.

[28] K.W. Böddeker, I.L. Gatfield, J. Jähnig, C. Schorm, Pervaporation at the vapor pressure limit: vanillin, J. Membr. Sci. 137 (1997) 155-158.

[29] C. Brazinha, D.S. Barbosa, J.G. Crespo, Sustainable recovery of pure natural vanillin from fermentation media in a single pervaporation step, Green Chem. 13 (2011) 2197-2203.

[30] G. Camera-Roda, V. Augugliaro, V. Loddo, L. Palmisano, Pervaporation membrane reactors, in: A. Basile (Ed.), Handbook of membrane reactors reactor types and industrial applications, Woodhead Publishing Series in Energy No vol. 2 in press, pp. 256.

[31] R.D. Hartley, E.C. Jones, Effect of ultraviolet light on substituted cinnamic acids and the estimation of their cis and trans isomers by gas chromatography, J. Chromatogr. 107 (1975) 213-218.

[32] K.W. Böddeker, G. Bengtson, H. Pingel, S. Dozel, Pervaporation of high boilers using heated membranes, Desalination 90 (1993) 249-257.

[33] G. Camera-Roda, F. Santarelli, M. Panico, Study and optimization of an annular photocatalytic slurry reactor, Photochem. Photobiol. Sci. 8 (2009) 712-718.

[34] C.S. Turchi, D.F. Ollis, Photocatalytic degradation of organic water contaminants: mechanisms involving hydroxyl radical attack, J. Catal. 122 (1990) 178-192.

[35] O. Levenspiel, Chemical Reaction Engineering, third ed., John Wiley \& Sons, New York, 1999.

[36] K. Mohan, R. Govind, Effect of temperature on equilibrium shift in reactors with a permselective wall, Ind. Eng. Chem. Res. 27 (1988) 2064-2070.

[37] P.K. Ten, R.W. Field, Organophilic pervaporation: an engineering science analysis of component transport and the classification of behaviour with reference to the effect of permeate pressure, Chem. Eng. Sci. 55 (2000) 14251445 . 